\title{
HIERARCHICAL STRUCTURE OF MALADAPTIVE PERSONALITY TRAITS IN OLDER ADULTS: JOINT FACTOR ANALYSIS OF THE PID-5 AND THE DAPP-BQ
}

\author{
Joke Van den Broeck, PhD, Leen Bastiaansen, $\mathrm{PhD}$, \\ Gina Rossi, PhD, Eva Dierckx, PhD, Barbara De Clercq, PhD, \\ and Joeri Hofmans, PhD
}

\begin{abstract}
In $D S M-5$, the categorical model and criteria for the 10 personality disorders included in DSM-IV will be reprinted in Section II. Moreover, an alternative dimensional classification model will appear in Section III. This alternative DSM-5 proposal for the diagnosis of a personality disorder is based on two fundamental criteria: impairments in personality functioning (Criterion A) and the presence of pathological personality traits (Criterion B). In the maladaptive trait model that has been developed to operationalize Criterion B, 25 pathological traits are organized according to five higher order dimensions. The current study focuses on the convergence of the proposed DSM- 5 trait model (as measured by the Personality Inventory for DSM-5 [PID-5]) with the Dimensional Assessment of Personality Pathology (DAPP) model (as measured by the Dimensional Assessment of Personality Pathology-Basic Questionnaire [DAPP-BQ]) in a sample of older people. A joint hierarchical factor analysis showed clear convergence between four PID-5 dimensions (Negative Affect, Detachment, Antagonism, Disinhibition) and conceptually similar DAPP-BQ components. Moreover, the PID-5 and the DAPP-BQ showed meaningful associations on different levels of their joint hierarchical factor structure. Methodological and theoretical implications of these initial results for the conceptualization of personality pathology are discussed.
\end{abstract}

The transition from the fourth edition of the Diagnostic and Statistical Manual of Mental Disorders (DSM-IV; American Psychiatric Association [APA], 1994) to the DSM-5 is currently being intensively prepared. According to the latest APA press release, the categorical model and criteria for the 10 personality disorders included in $D S M-I V$ will be maintained, whereas the alternative dimensional proposal will appear in Section III, aiming to encourage further research (http://dsmfacts.org/materials/

This article was accepted under the editorship of Robert F. Krueger and John Livesley.

Address correspondence to Joke Van den Broeck, Vrije Universiteit Brussel, Department of Clinical and Lifespan Psychology, Pleinlaan 2, B-1050 Brussels, Belgium; E-mail: Joke.Van. Den.Broeck@vub.ac.be 
american-psychiatric-association-board-of-trustees-approves-dsm-5/). In this proposal, the diagnosis of a personality disorder is based on five criteria (A through E) with two essential features of impairments in personality functioning (Criterion A) and the presence of pathological personality traits (Criterion B). Regarding the latter, a multidimensional maladaptive personality trait system has been proposed (Krueger, Derringer, Markon, Watson, \& Skodol, 2012). In this model, 25 primary traits are organized according to five higher order dimensions: Negative Affect, Detachment, Antagonism, Disinhibition, and Psychoticism. While constructing this trait model and its associated assessment instrument, the Personality Inventory for DSM-5 (PID-5; Krueger et al., 2012), the DSM-5 Personality and Personality Disorders Work Group relied on existing models of maladaptive personality traits, such as Harkness's Personality Psychopathology Five model (PSY-5; Harkness \& McNulty, 1994) and the Dimensional Assessment of Personality Pathology model (DAPP; Krueger et al., 2011; Livesley, Jackson, \& Schroeder, 1992).

Recently, the hierarchical structure of the DSM-5 personality trait model has been examined by applying Goldberg's (2006) "bass-ackward" analytic strategy on PID-5 data (Wright et al., 2012). At the fifth and final level of their analysis, Wright et al. (2012) replicated the PID-5 five-factor structure established by Krueger et al. (2012). At less differentiated levels of the hierarchy, the unfolding of the 25 proposed traits revealed structures that closely connected with common personality pathology models. At the second level, an Internalizing component (mainly marked by Depressivity, Anxiousness, and Withdrawal) and an Externalizing component (Manipulativeness, Risk Taking, and Attention Seeking) emerged from a general Personality Pathology factor. The Internalizing component then split into Detachment (Withdrawal, Anhedonia, and Restricted Affectivity) and Negative Affect (Emotional Lability, Anxiousness, and Perseveration), whereas the Externalizing component split into Antagonism (Manipulativeness, Grandiosity, and Callousness) and Disinhibition (Impulsivity, Risk Taking, and Distractibility). At the final level, a Psychoticism component (high loadings of Eccentricity, Perceptual Dysregulation, and Unusual Beliefs) emerged, which had no pronounced roots in any of the fourth level's components.

In the present study, we set out to investigate the convergent validity of the PID-5's hierarchical structure by means of a joint hierarchical factor analysis with the DAPP-BQ. Recently, Kushner, Quilty, Tackett, and Bagby (2011) delineated the hierarchical structure of the DAPP-BQ. At the sixth and lowest level, five factors showed conceptual resemblance to four PID-5 higher order dimensions: Emotional Dysregulation (Negative Affect), Inhibitedness (Detachment), Compulsivity (the opposite of Disinhibition), Dissocial Behavior/Disagreeable (Antagonism), Dissocial Behavior/Externalizing (Disinhibition), and Need for Approval. This last component, marked by high loadings of Insecure Attachment, Submissiveness, and Narcissism, has no clear counterpart in the PID-5 higher order domains, but 
conceptually connects to some lower order scales of Negative Affect (Separation Insecurity and Submissiveness) and Antagonism (Attention Seeking and Grandiosity). Although the hierarchical structures of the PID-5 and DAPP-BQ show considerable conceptual overlap, especially from Levels 1 through 4 , this has, to our knowledge, not yet been empirically tested. This study will do so by examining the joint hierarchical structure of the 25 proposed DSM-5 personality traits and the 18 DAPP dimensions. Because previous studies on the hierarchical structure of the DAPP-BQ (Kushner et al., 2011) and the PID-5 (Wright et al., 2012) focused on young adults and students, we extend this literature by focusing on an older adult sample. By doing so, we aim to contribute to the (sparse) research literature on the conceptualization of personality pathology in later life (e.g., Oltmanns \& Balsis, 2011). No a priori predictions were made about the exact unfolding of the joint PID-5/DAPP-BQ structure. However, we did expect the conceptually related PID-5 and DAPP-BQ traits to merge together in a formation parallel to their original unfolding. For example, at the fourth level of the hierarchy, we anticipated the PID-5 and DAPP-BQ scales to coincide with the established "Big Four" dimensions (Widiger \& Simonsen, 2005) as follows: PID-5 Negative Affect with DAPP-BQ Emotional Dysregulation, PID-5 Detachment with DAPP-BQ Inhibitedness, PID-5 Antagonism with DAPP-BQ Dissocial Behavior, and PID-5 Disinhibition with (reversed) DAPP-BQ Compulsivity. On the other hand, we expected unique traits (e.g., PID-5's Psychoticism-related traits) to emerge as a separate component in the unfolding procedure.

\section{METHOD}

\section{PARTICIPANTS AND PROCEDURE}

A total of 173 Dutch-speaking community-dwelling adults, recruited by undergraduate psychology students, participated. Ages ranged between 61 and 99 years $(M=72.72 ; S D=6.08)$, with $39.3 \%$ males. All participants provided a written informed consent.

\section{MEASURES}

PID-5. The Dutch authorized version of the Personality Inventory for DSM-5 (PID-5; De Clercq, De Fruyt, Mervielde, Krueger, \& Markon, 2011; Krueger et al., 2012) was used to measure the DSM-5 traits. The PID-5 has 25 primary lower order scales or facets that load onto five higher order personality pathology dimensions (Negative Affectivity, Detachment, Antagonism, Disinhibition, and Psychoticism). To examine whether the theorized PID-5 five-factor structure fits our data, we conducted exploratory factor analyses (EFA) in Mplus 7.0 (Muthén \& Muthén, 2012). The fivefactor solution was rotated toward the EFA five-factor solution reported by Krueger and colleagues (2012) using an oblique target rotation. Results 
revealed that a five-factor model fitted our data well $\left(\chi^{2} / d f=1.46\right.$, RMSEA $=$ $.05, \mathrm{SRMR}=.03, \mathrm{CFI}=.97, \mathrm{TLI}=.95)$. The standardized parameter estimates from this model are reported in Table 1 . The similarity of our factor solution with the target solution of Krueger and colleagues (2012) was assessed using congruency coefficients, yielding values of .88 for Negative affect, .95 for Detachment, .96 for Antagonism, .84 for Disinhibition, and .85 for Psychoticism. Descriptive statistics and alpha coefficients for the PID-5 scale scores are shown in Table 2. Current mean-level scores are broadly consistent with the data obtained by Krueger et al. (2012), although medium differences (i.e., Cohen's $d>.50$ ) were found for Grandiosity, Withdrawal, Intimacy Avoidance, and Separation Insecurity. Individuals in the current sample scored higher on the latter two, whereas individuals in the representative sample of Krueger et al. (2012) scored higher on Grandiosity and Withdrawal. Closer examination of the low alpha value for Suspiciousness [i.e., .25; with an average interitem correlation of .06 (range $=-.15$ to .49 )] revealed that this is mainly due to the two reversed scored items in this scale (i.e., i131 and i177). After deleting these two items from the scale, the alpha value increased to .54, with an average interitem correlation of .21 (range $=.00-.52$ ). Possibly these reverse-scored items share variance above and beyond the general factor of suspiciousness, making them qualitatively distinct from the other items in the scale and violating the assumption of local independence (e.g., Hop-

TABLE 1. Standardized Factor Loadings from the EFA Five-Factor Model of the PID-5

\begin{tabular}{|c|c|c|c|c|c|}
\hline Facet & $\begin{array}{c}\text { Negative } \\
\text { Affect }\end{array}$ & Detachment & Antagonism & Disinhibition & Psychoticism \\
\hline Emotional Lability & .59 & .08 & .16 & .03 & .20 \\
\hline Anxiousness & .71 & .28 & .03 & .12 & -.03 \\
\hline Separation Insecurity & .57 & .09 & .08 & .11 & .09 \\
\hline Perseveration & .42 & .12 & .28 & -.01 & .44 \\
\hline Restricted Affectivity & -.08 & .39 & .21 & -.05 & .18 \\
\hline Submissiveness & .28 & .21 & -.05 & .04 & .23 \\
\hline Withdrawal & -.16 & .70 & .09 & .04 & .19 \\
\hline Anhedonia & .08 & .62 & -.06 & .37 & -.03 \\
\hline Depressivity & .22 & .49 & -.12 & .28 & .38 \\
\hline Intimacy Avoidance & .01 & .48 & -.06 & .23 & -.05 \\
\hline Suspiciousness & .22 & .31 & .11 & .12 & .06 \\
\hline Manipulativeness & .03 & -.09 & .86 & .13 & -.01 \\
\hline Deceitfulness & .06 & .02 & .62 & .44 & .01 \\
\hline Grandiosity & -.13 & .03 & .65 & .08 & .25 \\
\hline Attention Seeking & .25 & -.12 & .55 & .13 & .26 \\
\hline Callousness & -.29 & .42 & .37 & .22 & .24 \\
\hline Hostility & .16 & .33 & .42 & .04 & .19 \\
\hline Impulsivity & .26 & .00 & .05 & .29 & .37 \\
\hline Irresponsibility & .05 & .00 & .22 & .62 & .17 \\
\hline Risk Taking & -.35 & -.22 & .22 & .10 & .46 \\
\hline Distractibility & .36 & .04 & -.05 & .43 & .43 \\
\hline Rigid Perfectionism & .40 & .20 & .39 & -.43 & .25 \\
\hline Eccentricity & -.00 & .05 & .19 & .32 & .62 \\
\hline \multicolumn{6}{|l|}{ Perceptual } \\
\hline Dysregulation & .16 & .10 & .03 & .49 & .49 \\
\hline Unusual Beliefs & .06 & .09 & .09 & .26 & .47 \\
\hline
\end{tabular}

Note. The highest loadings in each row are given in bold print. 
TABLE 2. Descriptive Statistics and Reliability for the PID5 Scales

\begin{tabular}{lcccrrrr}
\hline & $\alpha$ & Min & Max & M & SD & Skew & Kurt \\
\hline Submissiveness & .73 & .00 & 2.75 & 1.01 & .69 & .41 & -.50 \\
Depressivity & .88 & .00 & 2.36 & .45 & .47 & 1.75 & 3.67 \\
Separation Insecurity & .72 & .00 & 3.00 & 1.17 & .60 & .38 & -.17 \\
Perseveration & .74 & .00 & 2.33 & .83 & .51 & .42 & -.23 \\
Anxiousness & .84 & .00 & 3.00 & .95 & .63 & .82 & .14 \\
Emotional Lability & .85 & .00 & 3.00 & 1.04 & .69 & .31 & -.59 \\
Suspiciousness & .25 & .00 & 2.14 & 1.06 & .39 & .24 & .18 \\
Restricted Affectivity & .71 & .00 & 2.14 & .87 & .52 & .19 & -.75 \\
Withdrawal & .87 & .00 & 2.50 & .64 & .58 & .94 & .12 \\
Intimacy Avoidance & .68 & .00 & 2.67 & .99 & .62 & .47 & -.45 \\
Anhedonia & .75 & .00 & 2.63 & .73 & .49 & .81 & 1.01 \\
Manipulativeness & .82 & .00 & 3.00 & .58 & .63 & 1.34 & 1.74 \\
Deceitfulness & .84 & .00 & 2.60 & .48 & .50 & 1.85 & 3.97 \\
Hostility & .78 & .00 & 2.50 & .78 & .52 & .82 & .55 \\
Callousness & .77 & .00 & 1.73 & .41 & .39 & 1.50 & 2.03 \\
Attention Seeking & .86 & .00 & 2.75 & .68 & .61 & 1.02 & .59 \\
Grandiosity & .83 & .00 & 2.50 & .44 & .55 & 1.64 & 2.35 \\
Irresponsibility & .71 & .00 & 2.00 & .45 & .45 & 1.37 & 1.62 \\
Impulsivity & .72 & .00 & 2.67 & .87 & .58 & .70 & .19 \\
Distractibility & .84 & .00 & 2.33 & .80 & .60 & .63 & -.17 \\
Rigid Perfectionism & .85 & .00 & 2.50 & 1.10 & .61 & .24 & -.59 \\
Risk Taking & .74 & .00 & 2.43 & .96 & .42 & .34 & .31 \\
Eccentricity & .91 & .00 & 2.77 & .50 & .54 & 1.37 & 1.74 \\
Perceptual Dysregulation & .86 & .00 & 2.00 & .42 & .47 & 1.58 & 2.30 \\
Unusual Beliefs & .82 & .00 & 2.38 & .46 & .52 & 1.24 & .94 \\
\hline Note. N & & & & & & &
\end{tabular}

Note. $N=173$.

wood \& Donnellan, 2010). ${ }^{1}$ Mean-level comparisons across gender (not reported) revealed only one significant difference: men scored significantly higher than women on Risk Taking, $t(171)=4.586, p<.001$.

Dimensional Assessment of Personality Pathology-Basic Questionnaire (DAPP-BQ; Livesley \& Jackson, 2009). The Dutch translation of the DAPPBQ (van Kampen \& de Beurs, 2009) was used to measure personality pathology. It covers 18 personality disorder trait-based dimensions that are structured in four higher order factors (Emotional Dysregulation, Dissocial Behavior, Inhibition, and Compulsivity). Descriptive statistics and alpha coefficients for the DAPP-BQ scale scores are shown in Table 3. Current mean-level scores are broadly consistent with the normative data reported in the DAPP-BQ manual (Livesley \& Jackson, 2009, p. 27), although medium differences (i.e., Cohen's $d>.50$ ) were found for Stimulus Seeking, Insecure Attachment, and Intimacy Problems (with individuals in the current sample scoring higher on the latter two). A large difference (i.e., Cohen's $d>$.80) was found for Narcissism, for which a significant

1. We also conducted the joint hierarchical factor analyses with an abbreviated suspiciousness scale (i.e., without the two reverse-scored items). This had no impact on the unfolding of the hierarchical structure [i.e., identical one-, two-, three-, four-, and five-factor configurations were obtained as when the full-length suspiciousness scale was used and the factor loadings were also largely similar (the median change in factor loadings for the full versus abbreviated version was .14; range $=.00-.22$ )]. For this reason, and to ensure comparability with other PID-5 studies, we decided to retain the full-length suspiciousness scale for further analyses. 
TABLE 3. Descriptive Statistics and Reliability for the DAPP-BQ Scales

\begin{tabular}{lcccccrr}
\hline & $\alpha$ & Min & Max & $\boldsymbol{M}$ & SD & Skew & Kurt \\
\hline Suspiciousness & .85 & 1.00 & 4.36 & 2.07 & .67 & .63 & .38 \\
Affect Lability & .88 & 1.00 & 4.33 & 2.25 & .76 & .39 & -.59 \\
Cognitive Dysregulation & .90 & 1.00 & 4.00 & 1.74 & .66 & 1.10 & .90 \\
Identity Problems & .86 & 1.00 & 4.50 & 2.02 & .69 & 1.05 & .92 \\
Narcissism & .90 & 1.00 & 4.31 & 2.06 & .75 & .74 & .07 \\
Submissiveness & .85 & 1.00 & 4.50 & 2.28 & .64 & .52 & .43 \\
Insecure Attachment & .89 & 1.06 & 4.63 & 2.60 & .81 & .11 & -.72 \\
Oppositionality & .86 & 1.00 & 4.25 & 2.09 & .66 & .54 & -.06 \\
Low Affiliation & .90 & 1.00 & 4.25 & 2.10 & .77 & .50 & -.51 \\
Anxiousness & .92 & 1.00 & 4.73 & 2.26 & .84 & .58 & -.28 \\
Stimulus Seeking & .80 & 1.06 & 4.31 & 2.08 & .59 & .76 & .74 \\
Rejection & .89 & 1.00 & 4.38 & 2.28 & .73 & .40 & -.41 \\
Conduct Problems & .84 & 1.00 & 3.63 & 1.43 & .53 & 2.09 & 4.61 \\
Callousn & .84 & 1.00 & 3.56 & 1.84 & .60 & .84 & .05 \\
Restricted Expression & .68 & 1.44 & 3.75 & 2.66 & .52 & -.16 & -.61 \\
Intimacy Problems & .82 & 1.00 & 4.25 & 2.37 & .68 & .10 & -.45 \\
Compulsivity & .88 & 1.19 & 5.00 & 3.34 & .75 & -.26 & -.13 \\
Self-harm & .95 & 1.00 & 4.67 & 1.20 & .56 & 3.97 & 17.07 \\
\hline
\end{tabular}

Note. $N=173$.

higher mean-level score was found in the normative sample. Cronbach's alpha coefficients ranged from .68 (Restricted Expression) to .95 (Self-harm), which is also broadly consistent with normative data reported in the DAPP-BQ manual (Livesley \& Jackson, 2009). Mean-level comparisons across gender (not reported) revealed only one significant difference: women scored significantly higher than men on Intimacy Problems, $t(149)=$ $-3.982, p<.001$.

\section{STATISTICAL ANALYSES}

To examine the joint hierarchical structure of the PID-5 and the DAPP$\mathrm{BQ}$, the 25 primary DSM-5 traits and the 18 lower order dimensions of the DAPP-BQ were subjected to a series of varimax rotated principal component analyses (PCAs) with an increasing number of factors. To decide on the maximal number of factors, we relied on the minimum average partial (MAP) criterion, parallel analysis, prior theory and interpretability. Following Goldberg's (2006) "bass-ackward” method, we computed regressionbased factor scores on each level of the hierarchy, and these factor scores were subsequently correlated to compute path coefficients between the different hierarchical levels.

\section{RESULTS}

The MAP test suggested five factors. Parallel analysis indicated the existence of four factors; however, for the fifth one the difference between the actual and the random eigenvalues was only .01 (see Table 4). For these reasons, and because of a better interpretability, we decided to stop at the fifth level (a sixth factor in the six-factor solution was underidentified, with only two salient loadings of .58 and .42 for Suspiciousness and Intimacy Avoidance, respectively). In what follows, we will discuss each level of the 
TABLE 4. Parallel Analysis Based on Raw Data Permutations for 100 Random Data Sets

\begin{tabular}{ccc}
\hline Rank of Eigenvalues & $\begin{array}{c}\text { Eigenvalues of Sample } \\
\text { Correlation Matrix }\end{array}$ & $\begin{array}{c}\text { Mean Eigenvalues of Sample } \\
\text { Correlation Matrices Based } \\
\text { on Random Data Samples }\end{array}$ \\
\hline 1 & 19.61 & 2.11 \\
2 & 3.72 & 1.98 \\
3 & 3.33 & 1.88 \\
4 & 1.83 & 1.80 \\
5 & 1.72 & 1.73 \\
6 & 1.23 & 1.66 \\
7 & 1.12 & 1.59 \\
8 & 1.03 & 1.53 \\
9 & .91 & 1.47 \\
10 & .79 & 1.42 \\
\hline
\end{tabular}

Note. $N=173$.

joint hierarchical structure (see Figure $1^{2}$ ). The factor solutions used in the analysis of the PID-5/DAPP-BQ hierarchy are shown in Tables 5 and 6.

\section{LEVEL 1}

In the one-factor solution, all of the PID-5 and DAPP-BQ traits showed factor loadings larger than .40, with the exception of Intimacy Avoidance (.35) and Risk Taking (.20) for the PID-5 and Intimacy (.05) and Compulsivity (.24) for the DAPP-BQ. This component thus seemed to represent overall "Personality Pathology."

\section{LEVEL 2}

The general Personality Pathology component subdivided into two components, labeled "Internalizing/Emotional Dysregulation" and "Externalizing/Dissocial Behavior." The Internalizing/Emotional Dysregulation component was defined primarily by high loadings of the Anxiousness, Submissiveness, Depressivity, Emotional Lability, Separation Insecurity, and Anhedonia traits (PID-5) on the one hand, and by high loadings of the Anxious, Affective Lability, Submissiveness, Suspiciousness, Low Affiliation, and Identity problems dimensions (DAPP-BQ) on the other hand. Scales with salient loadings (>.40) on the Externalizing/Dissocial Behavior component were Grandiosity, Deceitfulness, Callousness, Manipulativeness, Attention Seeking (PID-5) and Callousness, Rejection, Narcisissm, and Stimulus Seeking (DAPP-BQ).

\section{LEVEL 3}

The Internalizing/Emotional Dysregulation component split into two subcomponents, "Detachment/Inhibitedness" and "Negative Affect/Emotion-

2. Path coefficients <.25 are not shown. 


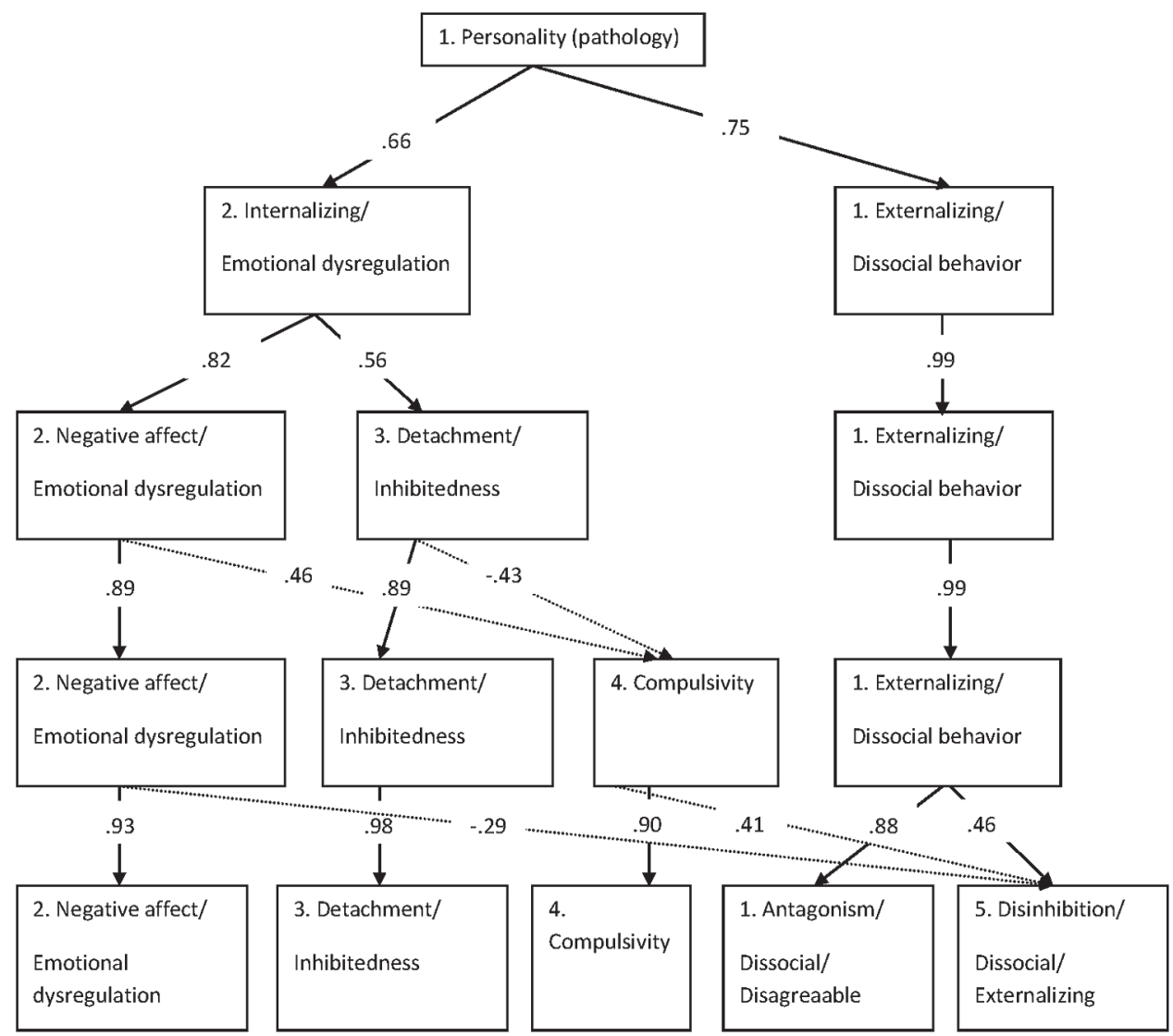

FIGURE 1. PID-5/DAPP-BQ joint hierarchical factor structure.

al Dysregulation," while the Externalizing/Dissocial Behavior component maintained its structure. PID-5 traits and DAPP-BQ dimensions that loaded highest on the Detachment/ Inhibitedness component were Withdrawal, Anhedonia, Intimacy Avoidance, Depressivity, and Restricted Affectivity (PID-5) and Identity Problems, Intimacy Problems, and Restricted Expression (DAPP-BQ). The component Negative Affect/Emotional Dysregulation was mainly marked by high loadings for Anxiousness, Emotional Lability, Separation Insecurity, and Perseveration of the PID-5 and Affective Lability, Submissiveness, Insecure Attachment, Low Affiliation, and Anxiousness of the DAPP-BQ.

\section{LEVEL 4}

The three components from the previous level were largely replicated, and a fourth component emerged. This new component was marked by PID-5 Rigid Perfectionism and DAPP-BQ Compulsivity, along with a negative loading of Intimacy Problems (DAPP-BQ). This component was labeled "Compulsivity." 
TABLE 5. Varimax-Rotated Factor Loadings for the One-, Two-, and Three-Factor Solutions

\begin{tabular}{|c|c|c|c|c|c|c|}
\hline \multirow[b]{2}{*}{ Facet } & \multirow{2}{*}{$\begin{array}{c}\text { One- } \\
\text { Factor } \\
\text { Model } \\
\text { I }\end{array}$} & \multicolumn{2}{|c|}{$\begin{array}{c}\text { Two-Factor } \\
\text { Model }\end{array}$} & \multicolumn{3}{|c|}{ Three-Factor Model } \\
\hline & & I. & II. & I. & II. & III. \\
\hline Submissiveness & .449 & .060 & .610 & -.020 & .495 & .362 \\
\hline Depressivity & .786 & .511 & .608 & .418 & .384 & .623 \\
\hline Separation Insecurity & .606 & .292 & .585 & .223 & .617 & .191 \\
\hline Perseveration & .816 & .613 & .538 & .549 & .641 & .148 \\
\hline Anxiousness & .700 & .277 & .743 & .188 & .756 & .262 \\
\hline Emotional Lability & .716 & .427 & .598 & .360 & .714 & .102 \\
\hline Suspiciousness & .493 & .424 & .265 & .389 & .296 & .129 \\
\hline Restricted Affectivity & .443 & .253 & .384 & .188 & .146 & .519 \\
\hline Withdrawal & .553 & .318 & .475 & .234 & .135 & .710 \\
\hline Intimacy Avoidance & .348 & .077 & .439 & -.003 & .050 & .716 \\
\hline Anhedonia & .602 & .301 & .568 & .208 & .248 & .705 \\
\hline Manipulativeness & .681 & .816 & .104 & .802 & .244 & .011 \\
\hline Deceitfulness & .741 & .833 & .176 & .798 & .159 & .267 \\
\hline Hostility & .782 & .731 & .354 & .684 & .427 & .162 \\
\hline Callousness & .643 & .710 & .168 & .669 & .009 & .443 \\
\hline Attention Seeking & .751 & .764 & .270 & .734 & .444 & -.002 \\
\hline Grandiosity & .671 & .806 & .100 & .787 & .172 & .108 \\
\hline Irresponsibility & .715 & .772 & .205 & .733 & .152 & .314 \\
\hline Impulsivity & .596 & .533 & .297 & .496 & .367 & .105 \\
\hline Distractibility & .845 & .657 & .533 & .577 & .411 & .486 \\
\hline Rigid Perfectionism & .575 & .324 & .502 & .273 & .674 & -.031 \\
\hline Risk Taking & .202 & .585 & -.358 & .629 & -.209 & -.191 \\
\hline Eccentricity & .761 & .796 & .248 & .752 & .198 & .327 \\
\hline Perceptual Dysregulation & .810 & .704 & .427 & .636 & .314 & .451 \\
\hline Unusual Beliefs & .659 & .648 & .261 & .605 & .211 & .298 \\
\hline Suspiciousness & .750 & .456 & .616 & .384 & .697 & .167 \\
\hline Affect Lability & .841 & .503 & .701 & .421 & .799 & .178 \\
\hline Cognitive Dysregulation & .876 & .543 & .708 & .442 & .554 & .557 \\
\hline Identity Problems & .784 & .373 & .762 & .261 & .506 & .685 \\
\hline Narcissisicm & .792 & .673 & .434 & .625 & .610 & .022 \\
\hline Submissiveness & .703 & .226 & .807 & .122 & .712 & .427 \\
\hline Insecure Attachment & .690 & .365 & .629 & .291 & .684 & .186 \\
\hline Oppositionality & .801 & .462 & .686 & .364 & .520 & .551 \\
\hline Low Affiliation & .808 & .392 & .777 & .283 & .592 & .589 \\
\hline Anxiousness & .789 & .286 & .869 & .177 & .818 & .395 \\
\hline Stimulus Seeking & .585 & .666 & .130 & .649 & .252 & .012 \\
\hline Rejection & .696 & .637 & .330 & .600 & .483 & .019 \\
\hline Conduct Problems & .751 & .719 & .320 & .662 & .200 & .434 \\
\hline Callousness & .818 & .735 & .404 & .677 & .403 & .288 \\
\hline Restricted Expression & .518 & .170 & .590 & .084 & .377 & .525 \\
\hline Intimacy Problems & .048 & -.079 & .163 & -.126 & -.235 & .613 \\
\hline Compulsivity & .239 & -.115 & .491 & -.156 & .678 & -.155 \\
\hline Self-harm & .548 & .594 & .154 & .552 & -.069 & .508 \\
\hline
\end{tabular}

Note. $N=173$. Factor loadings $\geq \mathrm{I} .40 \mathrm{I}$ are given in bold print.

\section{LEVEL 5}

At the fifth level of the hierarchy, the component Externalizing/Dissocial Behavior split to form two subcomponents, labeled "Antagonism/Disagreeable" and "Disinhibition/Externalizing." PID-5 Manipulativeness, Grandiosity, Hostility, Attention Seeking, Callousness, Deceitfulness and DAPP-BQ Rejection, Callousness, and Conduct Problems loaded strongest on the Antagonism/Disagreeable component. The Disinhibition/External- 
TABLE 6. Varimax-Rotated Factor Loadings for the Four- and Five-Factor Solutions

\begin{tabular}{|c|c|c|c|c|c|c|c|c|c|}
\hline \multirow[b]{2}{*}{ Facet } & \multicolumn{4}{|c|}{ Four-Factor Model } & \multicolumn{5}{|c|}{ Five-Factor Model } \\
\hline & I. & II. & III. & IV. & I. & II. & III. & IV. & V. \\
\hline Submissiveness & -.006 & .540 & .304 & .005 & -.107 & .625 & .260 & .101 & .030 \\
\hline Depressivity & .416 & .388 & .629 & .027 & .280 & .456 & .622 & .257 & .055 \\
\hline Separation Insecurity & .259 & .733 & .033 & -.068 & .303 & .715 & .042 & -.081 & .028 \\
\hline Perseveration & .547 & .573 & .174 & .277 & .537 & .536 & .162 & .186 & .316 \\
\hline Anxiousness & .206 & .781 & .196 & .123 & .308 & .694 & .187 & -.148 & .293 \\
\hline Emotional Lability & .381 & .746 & .017 & .120 & .360 & .756 & -.001 & .114 & .149 \\
\hline Suspiciousness & .370 & .188 & .226 & .261 & .441 & .082 & .234 & .028 & .348 \\
\hline Restricted Affectivity & .156 & .030 & .666 & .189 & .027 & .076 & .632 & .251 & .201 \\
\hline Withdrawal & .222 & .124 & .760 & -.023 & .178 & .119 & .773 & .051 & .090 \\
\hline Intimacy Avoidance & .022 & .215 & .589 & -.364 & .104 & .165 & .649 & -.304 & -.151 \\
\hline Anhedonia & .219 & .330 & .648 & -.161 & .246 & .295 & .683 & -.112 & .011 \\
\hline Manipulativeness & .812 & .253 & -.029 & .056 & .850 & .183 & .035 & .147 & .069 \\
\hline Deceitfulness & .823 & .261 & .152 & -.162 & .821 & .236 & .237 & .132 & -.134 \\
\hline Hostility & .678 & .361 & .202 & .219 & .649 & .329 & .214 & .245 & .228 \\
\hline sness & .654 & -.033 & .509 & .037 & .577 & -.035 & .552 & .264 & .044 \\
\hline Seeking & .740 & .416 & -.018 & .174 & .742 & .373 & .009 & .211 & .171 \\
\hline Gra & .797 & .192 & .068 & .006 & .840 & & 1 & .116 & .040 \\
\hline $\mathrm{Ir}$ & .757 & .257 & .201 & -.177 & & & & 33 & -.208 \\
\hline & .501 & .355 & .090 & .110 & .243 & .531 & .052 & .548 & -.069 \\
\hline lity & .597 & .492 & .395 & -.088 & .514 & .533 & .424 & .192 & -.053 \\
\hline tionism & .242 & .467 & .134 & .553 & .304 & .360 & .079 & .087 & .628 \\
\hline Risk Taking & .587 & -.400 & .005 & .321 & .315 & -.261 & -.023 & .720 & .035 \\
\hline Eccentricity & .749 & .184 & .339 & .049 & .538 & .298 & .350 & .510 & -.068 \\
\hline tual Dysregu & .652 & .384 & .376 & -.088 & .507 & .469 & .401 & .318 & -.118 \\
\hline al Beliefs & .600 & .187 & .320 & .072 & .370 & .323 & .310 & .510 & -.058 \\
\hline Suspiciousness & .364 & .553 & .274 & .423 & .450 & .430 & .249 & .022 & .553 \\
\hline Affect Lability & .424 & .743 & .184 & .290 & .403 & .726 & .149 & .163 & .340 \\
\hline Cognitive Dysregulation & .452 & .588 & .514 & .025 & .387 & .610 & .515 & .145 & .103 \\
\hline Identity Problems & .278 & .588 & .606 & -.091 & .265 & .585 & .618 & -.028 & .059 \\
\hline Narc & .628 & .557 & .025 & .257 & .625 & .518 & .024 & .202 & .271 \\
\hline & .137 & .744 & .369 & .078 & .081 & .783 & .329 & .066 & .158 \\
\hline ament & .311 & .719 & .108 & .0 & .336 & & & .0 & .181 \\
\hline Op & .371 & .543 & .522 & .035 & .304 & .568 & .517 & .133 & .112 \\
\hline Low Affiliation & .274 & .543 & .636 & .176 & .211 & .552 & .605 & .137 & .274 \\
\hline Ar & .177 & .773 & .409 & .250 & .147 & .771 & .355 & .083 & .349 \\
\hline Stimulus Seeking & .613 & .055 & .193 & .425 & .314 & .211 & .130 & .753 & .187 \\
\hline Rejection & .577 & .328 & .140 & .408 & .698 & .165 & .152 & .041 & .514 \\
\hline Conduct Problems & .667 & .227 & .411 & -.037 & .576 & .257 & .450 & .257 & -.034 \\
\hline Callousness & .680 & .392 & .280 & .099 & .727 & .309 & .323 & .077 & .192 \\
\hline Restricted Expression & .051 & .237 & .683 & .289 & .009 & .216 & .635 & .097 & .389 \\
\hline Intimacy Problems & -.082 & .035 & .389 & -.617 & .036 & -.009 & .486 & -.436 & -.412 \\
\hline Isivity & -.209 & .371 & .114 & .740 & -.118 & .243 & .004 & -.011 & .834 \\
\hline Self-harm & .548 & -.048 & .518 & -.107 & .399 & .027 & .555 & .311 & -.145 \\
\hline
\end{tabular}

Note. $N=173$. Factor loadings $\geq \mathrm{I} .40 \mathrm{I}$ are given in bold print.

izing component was strongly marked by PID-5 Impulsivity and Risk Taking and by DAPP-BQ Stimulus Seeking.

\section{DISCUSSION}

The goal of this study was to unravel the conceptual relations between the DSM-5 maladaptive personality traits and the DAPP-BQ's trait dimensions. The hierarchical structures of the PID-5 and DAPP-BQ coincided in expected ways from Levels 1 to 3, thereby mirroring the findings from both Wright et al. (2012) and Kushner et al. (2011). At the second level, the two 
broad Internalizing and Externalizing dimensions originated from the general Personality Pathology component, replicating the broadly recognized Internalizing-Externalizing dichotomy of psychopathology (e.g., Achenbach, 1966; Krueger, 2002). At the third level of the hierarchy, three dimensions emerged that link to the "Big-Three" model of temperament (i.e., Negative Affectivity/Emotional Dysregulation, Detachment/Inhibitedness, and Externalizing/Dissocial; Clark \& Watson, 2008; Wright et al., 2012).

The components at the fourth level of the hierarchy represented the established Big Four, with Negative Affect/Emotional Dysregulation, Externalizing/Dissocial Behavior, Detachment/Inhibitedness, and Compulsivity as major dimensions. Although we expected the PID-5 Disinhibition scales Irresponsibility, Impulsivity, Risk Taking, and Distractibility to represent the opposite pole of the Compulsivity component (thereby reproducing Widiger and Simonsen's, 2005, Constraint vs. Impulsivity bipolarity), they instead loaded primarily onto the Externalizing/Dissocial Behavior component, together with the PID-5's Antagonism and the DAPP-BQ's Dissocial Behavior indicators. Although this finding was rather unexpected, it is in line with the fact that the position of Disinhibition/Impulsivity versus Compulsivity has traditionally been a controversial issue. For example, in the initial DSM-5 proposal, Disinhibition and Compulsivity were considered separate structural components, with Disinhibition being conceptually linked to DAPP Dissocial Behavior (Krueger et al., 2011). Although both components were later unified into one bipolar domain (labeled "Disinhibition"; Krueger et al., 2012), the current findings connect with the initial proposal. However, future research is needed to fully explore this bipolarity issue, and whether this inconsistency is due to theoretical misspecifications or inadequate measurement.

In contrast to the study by Kushner et al. (2011), in which Compulsivity split off from the Dissocial component, the origins of Compulsivity in the present study were located in Negative Affect/Emotional Dysregulation and (reversely) in Detachment/Inhibitedness. One possible reason for this discrepancy may be that the Compulsivity component in the present study was somewhat broader (i.e., it included Rigid Perfectionism [PID-5], Compulsivity [DAPP-BQ], and Intimacy Problems [DAPP-BQ; negative loading]). Rigid Perfectionism is a (reversed) facet of Disinhibition in the PID-5, but it also shows considerable conceptual similarity to Perseveration, a facet of Negative Affect, which may explain its roots in this particular component. The negative loading of Intimacy Problems on Compulsivity is counterintuitive because it is assumed that the more structured and organized a person is, the more likely he or she is to be reserved and avoid intimacy. The reversal of this relationship in our study may therefore reveal a measurement bias; both the Intimacy Avoidance scale (PID-5) and the Intimacy Problems scale (DAPP-BQ) focus mainly on intimate relationships and sex, which may be a less valid indicator of intimacy in an older sample.

At the fifth and final level of the hierarchy, there was a bifurcation of the 
broad Externalizing dimension into Antagonism and Disinhibition. DAPPBQ's Rejection, Callousness, and Conduct Problems loaded highest on the former, and Stimulus Seeking on the latter. Counter to our expectations, a separate "Psychoticism" component, as present in the PID-5 five-factor structure (Krueger et al., 2012; Wright et al., 2012), did not emerge from our data. ${ }^{3}$ In contrast, the PID-5 Psychoticism scales loaded highest on the Antagonism (Eccentricity and Cognitive and Perceptual Dysregulation) and Disinhibition (Unusual Beliefs) components. At this point, it is important to stress that in our results the pathways of the PID-5 Psychoticism traits also differed from those in the Wright et al. (2012) study. In their study, Eccentricity and Cognitive and Perceptual Dysregulation originated from Detachment and Negative Affect, respectively (both derivatives of the Internalizing component), while Unusual Beliefs stemmed from the Externalizing component. Analogous joint factor-analytic research by Watson, Clark, and Chmielewski (2008) suggested that markers of "Oddity" (primarily defined by measures of dissociative tendencies and conceptually akin to PID-5's Psychoticism) formed a common dimension with Neuroticism in their two-, three-, and four-factor solutions. Clearly, more research is needed to examine this discrepancy more thoroughly. DAPPBQ's Cognitive Dysregulation did load highest onto Negative Affect/Emotional Dysregulation. This facet of the DAPP-BQ captures disorganized thinking and could therefore be expected to cluster together with the PID-5 Psychoticism scales. Hence, this finding reveals a conceptual difference between the PID-5 Psychoticism scales, focusing on odd thought processes in various sensory modalities and therefore referring more to schizotypal features, and the DAPP-BQ Cognitive Dysregulation scale, which rather marks transient thought disturbances and feelings of confusion resulting from extreme anxiousness and distress (Livesley \& Jackson, 2009). It is also important to note that the data were obtained from a community sample in which the prevalence of psychoticism features is likely to be low. This may explain why a separate psychoticism dimension was not observed in the current hierarchical structure.

Despite its methodological (i.e., joint hierarchical factor analysis) and conceptual (i.e., testing the hierarchical convergence of the PID-5 and the DAPP-BQ) contributions, our study is also subject to a number of limitations. First, the number of participants per variable was relatively small, impeding the generalizability of the current findings and making it difficult to distinguish real age effects from possible sample bias. Second, the low internal consistency of the PID-5's Suspiciousness scale in the current older sample warrants further investigation. Finally, further research

3. The three Psychoticism-related traits of Eccentricity, Cognitive Dysregulation, and Unusual Beliefs appeared as a separate component at the twelfth level of the joint hierarchical structure. Because one may wonder whether this is an artifact of factor analyzing the PID-5 together with the DAPP-BQ, in which Psychoticism content may be underrepresented, we also performed a hierarchical exploratory factor analysis on the PID-5 scores separately. In this analysis, no clear Psychoticism component emerged either. 
is needed to provide conclusive evidence regarding the position of Psychoticism features within a maladaptive trait model, the negative loading of Intimacy Problems on Compulsivity, and the structural relationship between Compulsivity and Disinhibition. Given these limitations and the exploratory nature of this study, the current findings should be given cautious consideration and warrant further exploration and replication. Nevertheless, the initial results of this study are valuable because they corroborate the idea of a common hierarchical structure underlying personality pathology (Krueger et al., 2011; Widiger \& Simonsen, 2005) and generally support the idea that the PID-5 allows us to capture this common structure. As such, our study adds to previous studies on the validity of the PID- 5 by not only showing that the DSM- 5 traits relate to the DAPPBQ's dimensions, but also demonstrating that the PID-5 and the DAPPBQ show meaningful associations on different levels of their hierarchical factor structures.

\section{REFERENCES}

Achenbach, T. M. (1966). The classification of children's psychiatric symptoms: A factor-analytic study. Psychological Monographs, 80(No. 615).

American Psychiatric Association. (1994). Diagnostic and statistical manual of mental disorders (4th ed.). Washington, DC: Author.

Clark, L. A., \& Watson, D. (2008). Temperament: An organizing paradigm for trait psychology. In O. P. John, R. W. Robins, \& L. A. Pervins (Eds.), Handbook of personality: Theory and research (3rd ed., pp. 265-286). New York: Guilford Press.

De Clercq, B., De Fruyt, F., Mervielde, I., Krueger, B., \& Markon, K. (2011). Dutch translation of the Personality Inventory for DSM-5 (PID-5).

Goldberg, L. R. (2006). Doing it all bass-ackwards: The development of hierarchical factor structures from the top down. Journal of Research in Personality, 40, 347-358.

Harkness, A. R., \& McNulty, J. L. (1994). The personality psychopathology five (PSY-5): Issue from the pages of a diagnostic manual instead of a dictionary. In S. Strack \& M. Lorr (Eds.), Differentiating normal and abnormal personality (pp. 291-315). New York: Springer.

Hopwood, C. J., \& Donnellan, M. B. (2010).
How should the internal structure of personality inventories be evaluated? Personality and Social Psychology Review, 14(3), 332-346.

Krueger, R. F. (2002). Psychometric perspectives on comorbidity. In J. E. Helzer \& J. J. Hudziak (Eds.), Defining psychopathology in the 21st century: DSM-V and beyond (pp. 41-54). Washington, DC: American Psychiatric Publishing.

Krueger, R. F., Derringer, J., Markon, K. E., Watson, D., \& Skodol, A. E. (2012). Initial construction of a maladaptive personality trait model and inventory for DSM-5. Psychological Medicine, 42(9), 1879-1890.

Krueger, R. F., Eaton, N. R., Clark, L. A., Watson, D., Markon, K. E., Derringer, J., et al. (2011). Deriving an empirical structure of personality pathology for DSM-5. Journal of Personality Disorders, 25(2), 170-191.

Kushner, S. C., Quilty, L. C., Tackett, J. L., \& Bagby, M. (2011). The hierarchical structure of the Dimensional Assessment of Personality Pathology (DAPPBQ). Journal of Personality Disorders, 25(4), 504-516.

Livesley, W. J., \& Jackson, D. N. (2009). DAPP-BQ: Dimensional Assessment of Personality Pathology-Basic Questionnaire. Technical manual. Port Huron, MI: Sigma Assessment Systems. 
Livesley, W. J., Jackson, D. N., \& Schroeder, M. L. (1992). Factorial structure of traits delineating personality disorders in clinical and general population samples. Journal of Abnormal Psychology, 101(3), 432-440.

Muthén, L. K., \& Muthén, B. O. (2012). Mplus user's guide (7th ed.). Los Angeles, CA: Muthén \& Muthén.

Oltmanns, T. F., \& Balsis, S. (2011). Personality disorders in later life: Questions about the measurement, course, and impact of disorders. Annual Review of Clinical Psychology, 27(7), 321-349.

Van Kampen, D., \& De Beurs, E. (2009). DAPP-BQ: Dimensionale assessment van persoonlijkheidspathologie inclusief screeningsversie [DAPP-BQ: Dimensional Assessment of Personality
Pathology-Basic Questionnaire and short form]. Amsterdam: Hogrefe.

Watson, D., Clark, L. A., \& Chmielewski, M. (2008). Structures of personality and their relevance to psychopathology: II. Further articulation of a comprehensive unified trait structure. Journal of Personality, 76(6), 1545-1586.

Widiger, T. A., \& Simonsen, E. (2005). Alternative dimensional models of personality disorder: Finding a common ground. Journal of Personality Disorders, 19(2), 110-130.

Wright, A. G. C., Thomas, K. M., Hopwood, C. J., Markon, K. E., Pincus, A. L., \& Krueger, R. F. (2012). The hierarchical structure of DSM-5 pathological personality traits. Journal of Abnormal Psychology, 121(4), 951-957. 